\title{
DETERMINATION OF STEERING WHEEL ANGLES DURING CAR ALIGNMENT BY IMAGE ANALYSIS METHODS
}

\author{
M. Mueller, T. Voegtle* \\ Institute of Photogrammetry and Remote Sensing (IPF), Karlsruhe Institute of Technology (KIT), Germany - \\ \{markus.mueller5, thomas.voegtle\}@ kit.edu
}

\author{
Commission V, WG V/1
}

KEY WORDS: Image analysis, image matching, quality assessment, industrial application

\begin{abstract}
:
Optical systems for automatic visual inspections are of increasing importance in the field of automation in the industrial domain. A new application is the determination of steering wheel angles during wheel track setting of the final inspection of car manufacturing. The camera has to be positioned outside the car to avoid interruptions of the processes and therefore, oblique images of the steering wheel must be acquired. Three different approaches of computer vision are considered in this paper, i.e. a 2D shape-based matching (by means of a plane to plane rectification of the oblique images and detection of a shape model with a particular rotation), a 3D shape-based matching approach (by means of a series of different perspectives of the spatial shape of the steering wheel derived from a CAD design model) and a point-to-point matching (by means of the extraction of significant elements (e.g. multifunctional buttons) of a steering wheel and a pairwise connection of these points to straight lines). The HALCON system (HALCON, 2016) was used for all software developments and necessary adaptions. As reference a mechanical balance with an accuracy of $0.1^{\circ}$ was used. The quality assessment was based on two different approaches, a laboratory test and a test during production process. In the laboratory a standard deviation of $\pm 0.035^{\circ}$ (2D shape-based matching), $\pm 0.12^{\circ}$ (3D approach) and $\pm 0.029^{\circ}$ (point-to-point matching) could be obtained. The field test of 291 measurements (27 cars with varying poses and angles of the steering wheel) results in a detection rate of $100 \%$ and $\pm 0.48^{\circ}$ (2D matching) and $\pm 0.24^{\circ}$ (point-to-point matching). Both methods also fulfil the request of real time processing (three measurements per second).
\end{abstract}

\section{INTRODUCTION}

In the industrial environment quality control has become an essential part of the production process. The aim of a $100 \%$ control in all stages can only be obtained by a comprehensive automation. Optical systems are of increasing importance in this wide-ranging field. Especially automatic visual inspections based on digital imagery allow contact-free real time measurements of high accuracy in dynamic production processes. Besides typical tasks like reading of labels, checks of completeness or the extraction of metric measures like length, distance, diameter etc. also more complex challenges can be met by image analysis. One of such new applications in this field is the determination of steering wheel angles during car alignment in the context of the final inspection of manufacturing. During wheel track setting the current steering wheel angle needs to be determined and subsequently aligned to a horizontal position (rotation angle $=0^{\circ}$ ). Until now this has been carried out by means of a mechanical steering wheel balance. In this research a steering wheel balance of manufacturer DSA (DSA, 2016) was used with an operation area of $\pm 20^{\circ}$. This balance delivers an accuracy of $0.1^{\circ}$ (Dürr, 2016a). Own investigations on the repeatability based on 100 measurements have confirmed an effective accuracy for small angles of approx. $\pm 0.03^{\circ}$ including the influences by slightly different manual positioning of the balance by an operator. Therefore, it is even better than the manufacturer's accuracy value of $0.1^{\circ}$ documented in the instructions. The requested accuracy for this application was defined by $\pm 0.2^{\circ}$. In the absence of alternative measuring techniques this balance has been taken as reference. The camera system has the advantage of contact-free measurement where the error component introduced by the manually positioning of the balance can be excluded. The camera has to be positioned outside the car to avoid any interruption of the dynamic manufacturing process and therefore, oblique images of the steering wheel are acquired through the opened side window of the car.

\section{RELATED WORK}

Many tasks in industrial environments can be solved with computer vision or machine vision systems (MVS). Image information supporting production lines or solving special tasks independently and thoroughly are getting more and more involved into the industrial processes of many manufacturers. Nowadays, tasks like completeness checks (Haniff et al., 2011), inspections (Molleda et al., 2013), (pre-)selections of construction elements (Rodrigues et al., 2012) or quality tests (Gunasekaran, 1996) are already fulfilled using the potential of robust machine vision systems operating in real time. The

\footnotetext{
* Corresponding author
} 
required parameters are directly determined by the MVS and then transferred for further processing or evaluation.

In this paper image analysis methods like shape-based matching or image rectification are used (HALCON, 2015a). Shape-based matching can be performed using two- or three-dimensional approaches. 2D methods can be found in (Haniff et al., 2011), (Xu et al., 2008) or (Fan et al., 2014) and are, in principle, suitable to achieve sub-pixel accuracies. 3D approaches determining the pose of an object like reported in (Ulrich et al., 2009) or (Reinbacher et al., 2010) have advantages over 2D methods but may lead to insufficient results according to real time capability or accuracy for specific tasks. Cars need to pass several alignment and initial operation processes before their delivery. One of these alignment steps is the adjustment of the chassis. During the setting of the front suspension the so-called steering angle needs to be aligned to the front axle. Usually the determination of this steering angle is carried out by means of a mechanical steering wheel balance at leading automotive manufacturers (Figure 1).

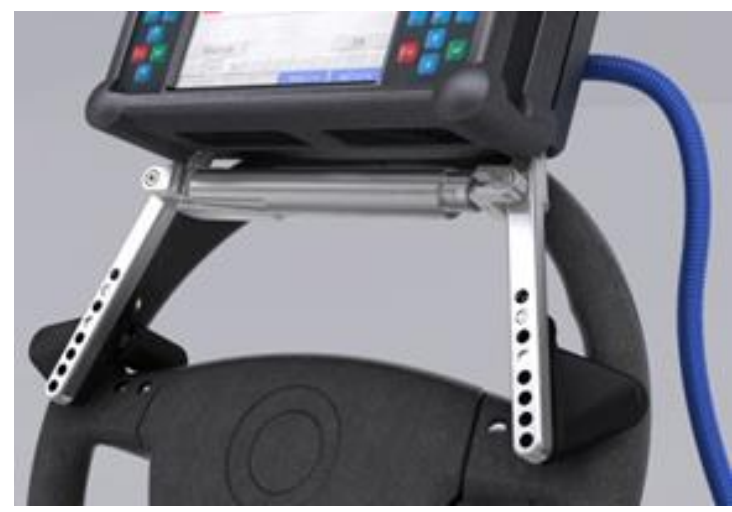

Figure 1. Attached steering wheel balance (DSA, 2016)

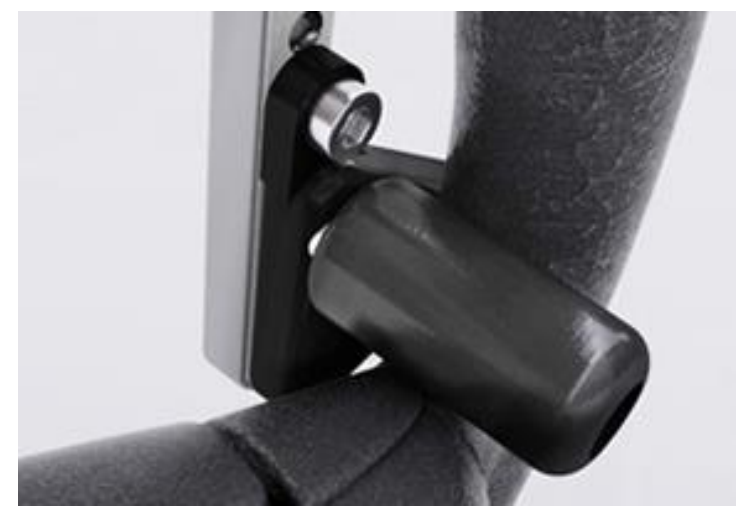

Figure 2. Tactile supporting point of the balance (DSA, 2016)

This balance gets attached on the steering wheel manually and measures the current rotation angle of the steering wheel to the horizontal. Steering wheel balances are getting distributed by companies like Dürr AG (Dürr, 2016b) or DSA (DSA, 2016). Duerr AG specifies its steering wheel balance named $x$-tronic balance within a measuring accuracy of $0.1^{\circ}$ (Dürr, 2016a). Additional to this uncertainty, a dimensional tolerance has to be added caused by slight deviations of the (tactile) attachment (Figure 2).

\section{DATA ACQUISITION AND PRE-PROCESSING}

Compared to mechanical steering wheel balances camera based vision systems have two main advantages. On the one hand additional error influences by manual positioning can be avoided due to contact-free measurements, on the other hand the production process has not to be interrupted. For this vision system the industrial camera UI-5370 (IDS, 2016) was applied. Its features are sampled in Table 1.

\begin{tabular}{|l|l|}
\hline Camera & UI-5370 (IDS) \\
\hline sensor type & CMOS \\
\hline size of sensor elements & $5.5 \mu \mathrm{m}$ \\
\hline sensor size & $2048 \times 2048$ pixels \\
\hline radiometric resolution & $12 \mathrm{bit} / \mathrm{pixel}$ \\
\hline
\end{tabular}

Table 1. Data of the industrial camera UI-5370

But as described above, the images of the steering wheel have to be acquired from outside the car in an oblique viewing direction through the opened side window. Common distances of approx. $2 \mathrm{~m}$ between steering wheel and camera can be realized which results in pixel sizes of 0.10 to $0.15 \mathrm{~mm}$ on the object. First test series have shown that the common illumination in real production environments is normally not sufficient for this task. Therefore, special lighting devices (e.g. ring-flashes) had to be applied for a more uniform illumination and suitable image contents. The real time requirements of the producer are defined as three measurements per second.

As additional input data for the subsequent analysis of these oblique images CAD models of the steering wheel types used in this investigation are available. A fixed local coordinate system has been defined for the steering wheels, where the $\mathrm{x}$-axis is horizontal, the y-axis is vertical and the $\mathrm{z}$-axis is orthogonal to these two axes (all related to the CAD construction plan).

The data acquisition has been carried out in two parts. On the one hand several image series were generated in the laboratory to develop and investigate different analysis approaches, on the other hand images were recorded directly in the real production environment to evaluate and improve suitable methods.

A first image series aimed to the determination of the repeat accuracy. A steering wheel was (mechanically) fixed in its basic pose and captured by 100 images resulting in a repeat accuracy of $\pm 0.0007^{\circ}$ which has confirmed the extremely high quality and the potential of our vision system (cf. repeat accuracy of the mechanical steering wheel balance was $\pm 0.03^{\circ}$ ). To generate enough test material for development and evaluation of appropriate analysis methods, different rotation angles (mechanical balance as reference) and heights of the steering wheel (for several types of cars the steering wheel can be moved in height about $10 \mathrm{~cm}$ ) have been captured by the camera in that oblique viewing direction according to the perspective in the production environment. In the domain of $\pm 5^{\circ}$ the rotation angle has been incremented by steps of $1^{\circ}$, in the remaining domain of $\pm 10^{\circ}$ in increments of $2.5^{\circ}$. This acquisition constellation was repeated for each of three different heights, the middle position and $+5 \mathrm{~cm}$ and $-5 \mathrm{~cm}$ respectively. Therefore, 15 images have been stored for each height position, which overall results in 45 images. 
In the real production process a first image series was acquired with the camera mounted on an already existing pole (used for other tasks). It could be recognized from the first results that the pole was affected by concussions caused by the surrounding machines. Therefore, a second image series was captured where the camera was fixed to a static construction component of the building. Additionally, a ring illumination around the camera lens was used to guarantee uniform and constant lighting conditions. This series comprises 291 images of 27 cars of two similar steering wheel types, i.e. approx. 10 measurements per car in a time period of $3 \mathrm{sec}$., which fulfils the real time request of three measurements per second for this application.

\section{METHODOLOGY}

Three different approaches were developed to solve the problem of a real time determination of steering angles during the car alignment procedure in industrial environments. A calibrated camera is a prerequisite for any of our three applied approaches. Therefore, the machine vision system consists of a calibrated camera, a computer and an illumination unit as a constant configuration for all evaluations. In consequence, the methods only differ in their software-based implementation.

\subsection{D Shape-based Matching}

The first implementation was realized by a 2D shape-based matching using HALCON routines (HALCON, 2015b). Unlike other methods this technique does not operate with grey values directly or correlation-based approaches but uses extracted contours of an object to search for any number of instances of this object in arbitrary images. In principle, the reference contours can be extracted in an image containing the specified object in a pre-defined rotation. These contours are stored in different image pyramid levels and rotations in the reference plane in a user-defined range (stepwise in small increments) as reference. This is called a $2 \mathrm{D}$ shape-model. An object can be searched using the provided shape-model with its rotations and scales. An instance of an object can only be recognized if it appears in the provided rotation- and scale-range, otherwise it will not be detected. After a coarse detection of possible instances fitting the quested shape, based on a modified generalized Hough transform (Ulrich et al., 2003), a pose refinement is performed. For our specific application we make use of this 2D shape-based matching method using the multifunctional buttons of the steering wheels as distinctive marks serving as such a shape-model (cf. Figure 3).
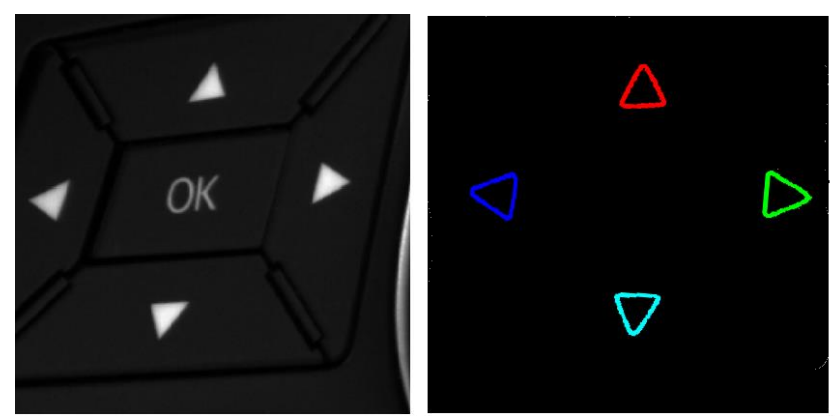

Figure 3. Creating a shape-model from reference image. Left: image containing a field of multifunctional buttons. Right: extracted contours of created shape-model
Supposing an orthogonal view of the steering wheel the rotation of an arbitrary element is equivalent to a rotation of the steering wheel itself. Therefore, the rotation of the multifunctional buttons can be used for the determination of the steering angle. Due to oblique viewing directions through the side window an orthogonal orientation of the camera to the steering wheel is not realizable. Because of the resulting perspective distortions a 2D-rotation of the steering wheel (and its components) does not correspond to its rotation in the image. To overcome this problem a geometric rectification is applied, transforming an oblique image into an orthogonal view by means of a defined reference plane. Then the rotation of a component of the wheel in the rectified image is equivalent to the requested rotation of the steering wheel itself. However, for the absolute determination of the rotation of a steering wheel, we make use of a reference image showing a horizontal aligned steering wheel (angle $=0^{\circ}$ ). The rotation of the extracted shape model in that image will be used as initial value $\Phi_{\text {Ref. }}$ Then, in subsequently acquired images we can compute the difference between the current steering wheel rotation $\Phi_{\mathrm{i}}$ in image $i$ and the reference rotation $\Phi_{\text {Ref }}$ to obtain the required current steering wheel angle $\lambda_{\mathrm{i}}$

$$
\lambda_{\mathrm{i}}=\Phi_{\mathrm{i}}-\Phi_{\mathrm{Ref}}
$$

\subsection{D Shape-based Matching}

Another option for solving this task is given by a 3D shapebased matching (HALCON, 2015c). This approach is able to deliver a 3D pose of an object by means of a series of slightly different views of the 3D CAD construction plan by a virtual monocular camera (Ulrich et al., 2009). This series of virtual images covers a user-defined range of possible viewing angles around the main viewing direction as well as different distances of the camera (both related to the subsequent application) in small increments (Figure 4). Additionally, for each of these camera poses a series of views with different rotation angles is created as a basis for the further determination of the steering angle. The total of all images is combined to a 3D shape model.

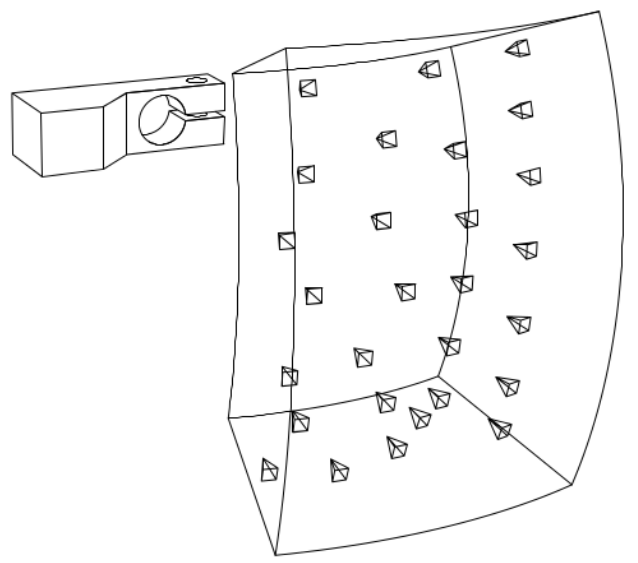

Figure 4. Several model views from different camera positions and orientations in a user-specified area pointing to the origin of the object (Ulrich et al., 2009)

Before the determination of the requested steering angle in the production process can be started, a reference image of a horizontal aligned steering wheel (by means of the mechanical 
balance) has to be acquired. Subsequently, in a current image an edge extraction is carried out. These edges are compared to the contours of the 3D shape model by a best fit procedure (matching) to obtain the pose of the steering wheel related to the camera coordinate system. Now, the steering angle can be calculated by the difference to the reference data. Similar to the 2D approach explained above a pose refinement is processed after the determination of the coarse view respectively coarse pose.

Complex CAD models containing a huge number of geometric primitives may lead to insufficient processing times. Therefore, these CAD models should be reduced to the main significant and distinctive elements to guarantee real time processing. Nevertheless, spatial expanded elements will lead to more robust results concerning the determination of the rotational angle. A suitable choice is the use of multifunctional buttons spatially far apart (Figure 5).
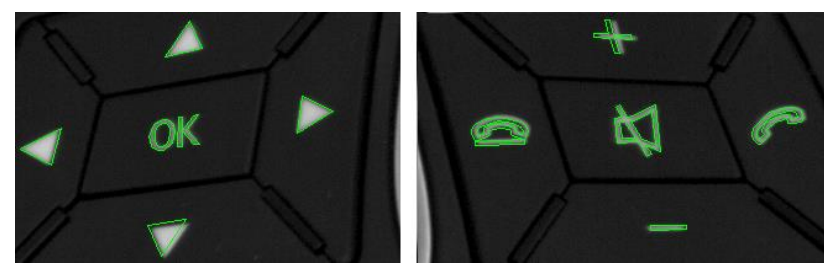

Figure 5. Matching results of the CAD model (green)

\subsection{Point-to-Point Matching}

For the third approach a procedure similar to the 2D shapebased method is considered (Section 4.1). In contrast to this method described above the pixel-positions of at least two wheel elements (e.g. multifunctional buttons) are used to calculate the steering angle. Again a rectification is applied to generate a transformation into a frontal orthogonal view. Also, a reference image containing a steering wheel with a known rotation (e.g. $0^{\circ}$ ) is captured and rectified. In this reference image at least one pair of points is extracted. Therefore, the connection between these points (visualized by a straight line in Figure 6) has a specific (reference) slope $\Phi_{\text {Ref. In each }}$ subsequent image $i$ the same pair of points is extracted to calculate their slope $\Phi_{\mathrm{i}}$. Now, the steering angle $\lambda$ can be determined by the difference between the slopes $\Phi_{\text {Ref }}$ and $\Phi_{\mathrm{i}}$ (cf. Equation (1)). To enhance the robustness of this approach, additional pairs of points may be added. For instance, in Figure 13 six pairs of points based on those significant elements have been selected.
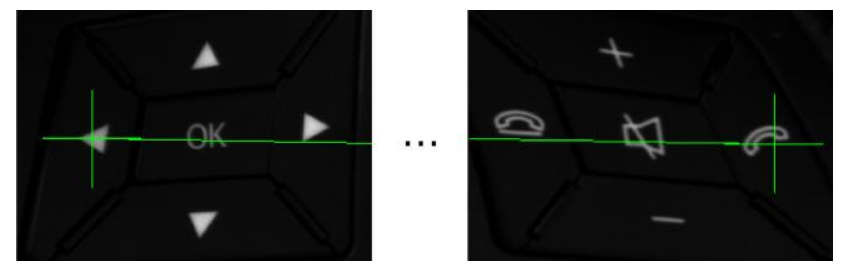

Figure 6. Two extracted points (one in the left field of multifunctional buttons and one in the right field) and their connecting straight line

\section{RESULTS}

The operational capability of the introduced methods was initially tested in a laboratory environment. Subsequently, this machine vision system was integrated into the production process to obtain real world data.

\subsection{Laboratory Tests}

5.1.1 2D Shape-based Matching: For the 2D shape-based matching approach the multifunctional buttons on the left side of the steering wheel (Figure 3) were used as distinctive elements to create a shape model. The steering angle was computed in 45 images of the same steering wheel in different rotations (from $-10^{\circ}$ to $10^{\circ}$ ) and poses (low, mid and high). The results compared to the mechanical balance are plotted in Figure 7. For large angles - especially $\pm 10^{\circ}$ - the differences increase for all three positions, but by far do not reach the defined threshold of accuracy $\left(0.2^{\circ}\right)$. The position respectively pose of the steering wheel slightly affects the determination throughout the whole angular range. The standard deviation according to the reference measurements of the mechanical balance is $\pm 0.035^{\circ}$.

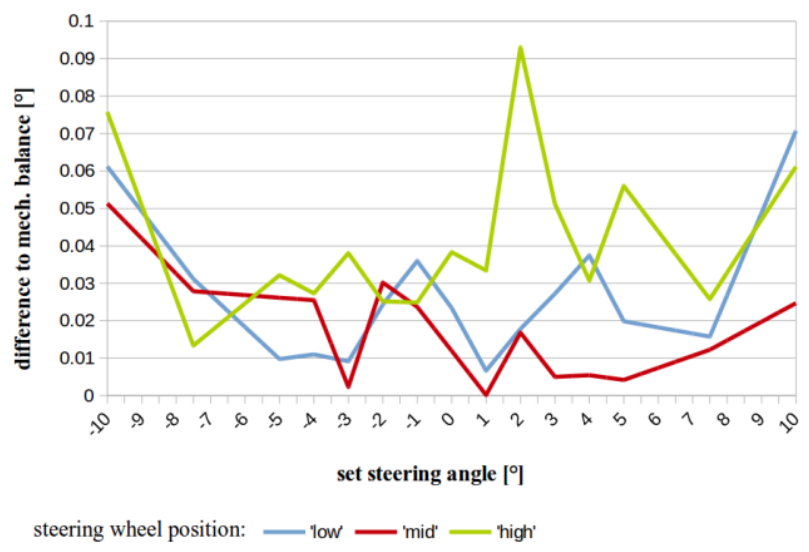

Figure 7. Differences of determined steering angles related to the mechanical balance using $2 \mathrm{D}$ shape-based matching

5.1.2 3D Shape-based Matching: In tests using different distinctive elements of the CAD model to compute the steering angle, gross differences to the mechanical balance occurred (up to 8 pixels, Figure 5). A deviation of such amount leads to a determination error of approx. $0.4^{\circ}$. Investigations confirmed such discrepancies between the CAD model and the manufactured steering wheel elements. This is known as dimensional tolerances. However, these tolerances lead to unsatisfying matching results. Finally, the best results could be obtained using the manufacturer's emblem in the centre of the steering wheel. Figure 8 illustrates the results of a series of measurements. The standard deviation is $\pm 0.41^{\circ}$. However, ignoring the two gross errors at $-7.5^{\circ}$ and $-2^{\circ}$, the standard deviation decreases to $\pm 0.12^{\circ}$. A trend of increasing absolute differences for large angles is visible. The threshold of $0.2^{\circ}$ is exceeded several times. 


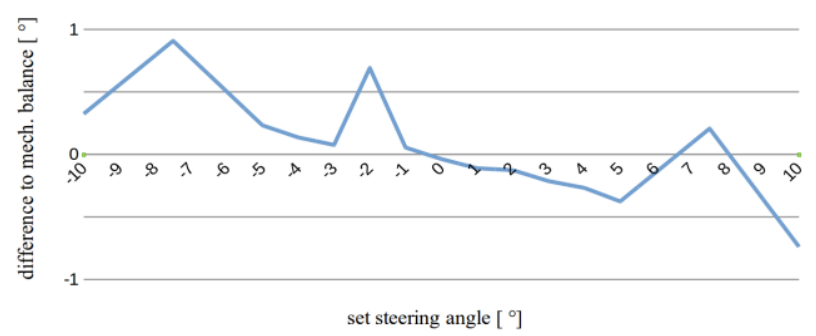

Figure 8. Differences of determined steering angles related to the mechanical balance using 3D shape-based matching

5.1.3 Point-to-Point Matching: The point-to-point matching was evaluated analogue to the $2 \mathrm{D}$ shape-based matching. The pair of points consists of one element (button) from the left side and one from the right side of the steering wheel as shown in Figure 6. The steering angle was computed in 45 images containing the same steering wheel in different rotations and poses. The results compared to the mechanical balance are shown in Figure 9. These are similar to those illustrated in Figure 7. However, the overall accuracy is slightly better resulting in a standard deviation of $\pm 0.029^{\circ}$. The obtained values do not exceed $0.1^{\circ}$ and lie far below the threshold of $0.2^{\circ}$.

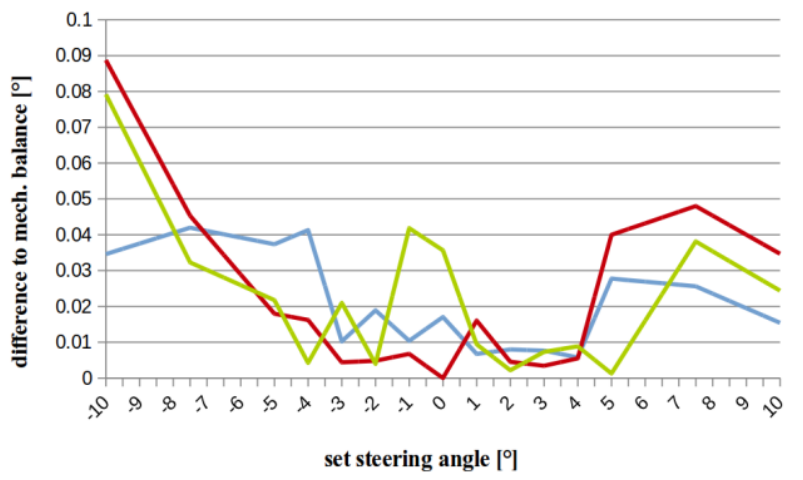

steering wheel position: —— "low' — 'mid" —— "high'

Figure 9. Differences of determined steering angles related to the mechanical balance using point-to-point matching

\subsection{Field Tests}

To evaluate the approaches using real data from industrial environment, the machine vision system was integrated into the production process. A reference image (steering angle $=0^{\circ}$ ) and the steering wheels pose definition need to be determined once after the camera and all parts of the machine vision system are installed at the production site. Hence the 3D matching did not deliver sufficient results - even more gross errors occurred - it was excluded in the further analysis.

To confirm the sensitivity of such camera systems, Figure 10 shows how mechanical influences during the production process affect the measurements of the vision system. At the beginning the camera was mounted at a relatively thin pole which tended to vibrations caused by concussions of the surrounding machines. After a stop of the machines the camera (and therefore the measurements) stabilizes beyond number 42 (Figure 10). Thereafter the camera was positioned on a static pole without physical connection to the production platform. For this reason, further problems had been avoided.

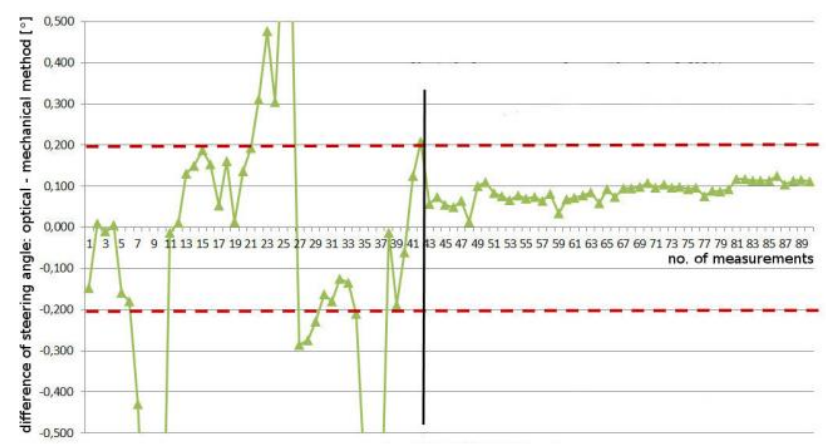

Figure 10. Differences of optical and mechanical determined steering angles. Up to measurement 42 (vertical line) the effect of concussions caused by the production environment is clearly visible. After measurement 42 , the camera was stabilized.

5.2.1 2D Shape-based Matching: The results of the 2D approach are visualized in Figure 11. 291 measurements including 27 different vehicles are plotted in the histogram of the obtained differences to the mechanical steering wheel balance. The red line indicates the accumulated differences. Only $21,4 \%$ of the measurements lie beneath the threshold of $0.2^{\circ}$. A wide variation of differences is clearly visible. The detection rate is $100 \%$, i.e. for each image the distinctive elements could be automatically recognized and the steering angle could be calculated. The standard deviation for the plotted differences is $\pm 0.48^{\circ}$.

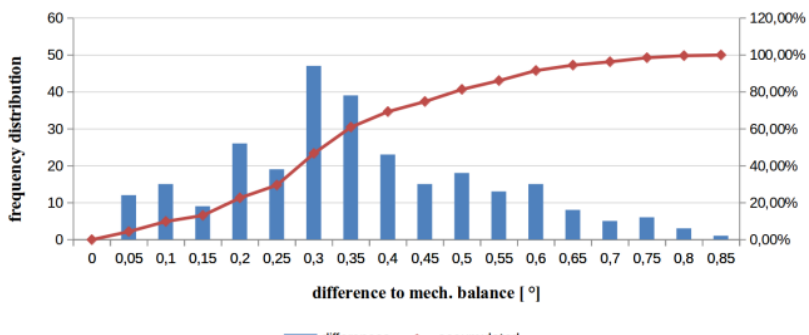

Figure 11. Histogram of the differences between the optical 2D shape-based and the mechanical measurement method

5.2.2 Point-to-Point Matching: The point-to-point approach was analyzed based on the identical image series of 27 cars. Here 261 measurements were carried out. The difference of 30 measurements to the shape-based method results from partial occlusions, hence the point-to-point matching uses the left and the right field of multifunctional buttons which both must be visible in the same image. These occlusions are caused by the human operator at the beginning of a series of functional checks during the car alignment. However, multiple determinations of the steering angle could be obtained for each car. Figure 12 visualizes the corresponding histogram for the point-to-point matching results, again calculated by the differences to the mechanical balance. These differences obtained by the point-topoint matching show significantly more small values compared to the $2 \mathrm{D}$ shape-based matching. This leads to a better accuracy and therefore to a standard deviation of $\pm 0.24^{\circ}$. Furthermore, $61.5 \%$ of the differences lie below the limit of $0.2^{\circ}$ and even $80.4 \%$ do not exceed the value of $0.25^{\circ}$. 


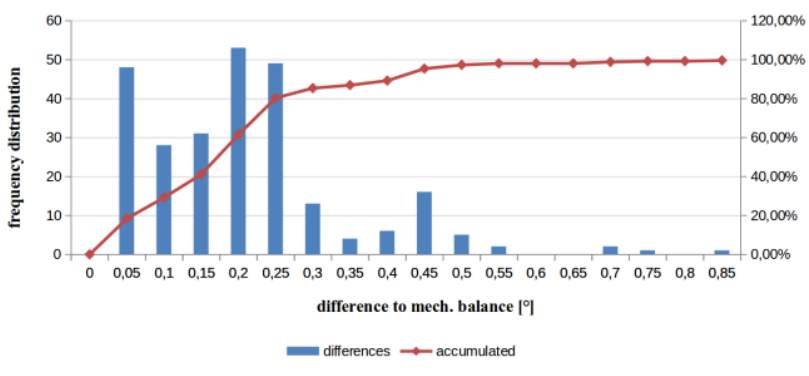

Figure 12. Histogram of the differences between the optical point-to-point and the mechanical measurement method

\subsection{Real Time Capability}

To verify the real time capability of the applied approaches Table 2 shows the processing time for the determination of the steering angle of ten subsequent measurements in production environment. The time for image acquisition is included and took approximately $106 \mathrm{msec}$ per image. As can be seen clearly the 2D method (144 msec) and the point-to-point approach (136 msec) reach real time capability. The 3D shape-based matching (348 msec) exceeds the threshold slightly. However, by decreasing the search space of the 3D approach, a faster computation than presented in Table 2 could be obtained. Nevertheless, according to the insufficient accuracies of the 3D shape-based matching an optimization was not intended.

\begin{tabular}{c|c|c|c} 
No. & $\begin{array}{c}2 \mathrm{D} \text { shape-based } \\
{[\mathrm{msec}]}\end{array}$ & $\begin{array}{c}\text { 3D shape-based } \\
{[\mathrm{msec}]}\end{array}$ & $\begin{array}{c}\text { point-to-point } \\
{[\mathrm{msec}]}\end{array}$ \\
\hline 1 & 145 & 400 & 142 \\
\hline 2 & 144 & 377 & 138 \\
\hline 3 & 145 & 362 & 137 \\
\hline 4 & 141 & 298 & 133 \\
\hline 5 & 142 & 318 & 134 \\
\hline 6 & 142 & 323 & 133 \\
\hline 7 & 143 & 337 & 134 \\
\hline 8 & 144 & 346 & 135 \\
\hline 9 & 145 & 356 & 136 \\
\hline 10 & 146 & 362 & 136 \\
\hline \hline average & $\mathbf{1 4 4}$ & $\mathbf{3 4 8}$ & $\mathbf{1 3 6}$
\end{tabular}

Table 2. Overview of total processing times of the introduced methods including the time for image acquisition $(106 \mathrm{msec})$

\section{DISCUSSION}

For determination of all results presented in the preceding chapter a mechanical balance was used as reference. Therefore, it has to be taken into account that those balances have also a certain (limited) accuracy, i.e. the differences between the steering wheel angles extracted by the computer vision system and the mechanical balance contain also a component caused by the balance itself. This should be regarded for the assessment of the results, especially when taking into account the high interior measurement accuracy of the computer vision system confirmed during the laboratory research.
Comparing the results of the laboratory tests of the three approaches it is obvious that the 3D shape-based matching has proved to be not sufficient for this specific task concerning robustness (gross errors occurred) and accuracy $\left( \pm 0.12^{\circ}\right)$. The reasons can be found in the (limited) manufacturing accuracy of the multifunctional buttons (given by $\pm 0.2 \mathrm{~mm}$ ) which prevents an optimal fit of the CAD construction plan. The 2D shapebased matching has proved a high robustness, i.e. a detection rate of $100 \%$ and an accuracy of $\pm 0.035^{\circ}$ due to several larger deviations. Similar results could be obtained by the point-topoint matching. The high robustness of $100 \%$ and the accuracy of the steering angle of $\pm 0.029^{\circ}$ are comparable to the 2D method which confirms the high quality potential of these image analysis approaches.

Even if defined and stable conditions for image acquisition could be realized in both environments (stable camera mounting, constant and uniform lighting conditions etc.) the results of the laboratory tests are significantly better compared to the field tests. In the laboratory for all investigations only one identical steering wheel was used. Therefore, positional deviations of the distinctive elements (multifunctional buttons) could be avoided. In contrast, in production environment 27 different cars have been analyzed with the same steering wheel type but certain manufacturing tolerances $( \pm 0.2 \mathrm{~mm})$. These slightly different steering wheel geometries lead to uncertainties in the determination of the steering angle. Another reason is the new manual attachment of the balance for each car. So the accuracy of the 2D shape-based matching is significantly reduced to $\pm 0.48^{\circ}$ and for the point-to-point matching to $\pm 0.24^{\circ}$, even if the determination of the position of the extracted features (multifunctional buttons) is carried out in a high accuracy (sub-pixel domain). For the 2D shape-based matching the entire field of these multifunctional buttons (on the left or right side of the steering wheel) is used as pattern which leads to a reduced fitting accuracy due to these tolerances. For the point-to-point matching individual buttons on both sides are extracted separately resulting in larger distances and therefore, in more stable baselines and finally in smaller deviations of the determined steering angle.

Another main aspect of data acquisition in real production environments concerns the extremely stable mounting of the camera. It could be verified that the computer vision system has a high sensitivity due to exterior mechanical influences. For instance, the relatively thin metal pole used as mounting device in first field tests tended to vibrations (caused by concussions of other machines in the manufacturing hall) introducing effects significantly larger than the measurement accuracy (cf. Figure 10, up to measurement 42). After termination of these exterior influences, realistic values with slight deviations could be obtained.

Concerning the processing time, the point-to-point matching is the fastest method $(30 \mathrm{msec})$, followed by the 2D shape-based matching $(38 \mathrm{msec})$ and the 3D shape-based matching (242 $\mathrm{msec}$ ). Additionally, the time for image acquisition of approx. $106 \mathrm{msec}$ has to be taken into account. In total, the 3D shapebased matching is the only method, which does not fulfil the requirement of real time processing, i.e. 3 measurements per second $(=333 \mathrm{msec})$, whereas both described $2 \mathrm{D}$ approaches meet the real time condition.

Overall, the point-to-point matching has proved to be the fastest and most accurate method which approximately reaches the defined accuracy threshold of $\pm 0.2^{\circ}$ in the production 
environment. An improvement can be achieved by using multiple lines between distinctive elements (Figure 13). Regarding additionally the uncertainty of the mechanical balance itself the obtained accuracy is acceptable.

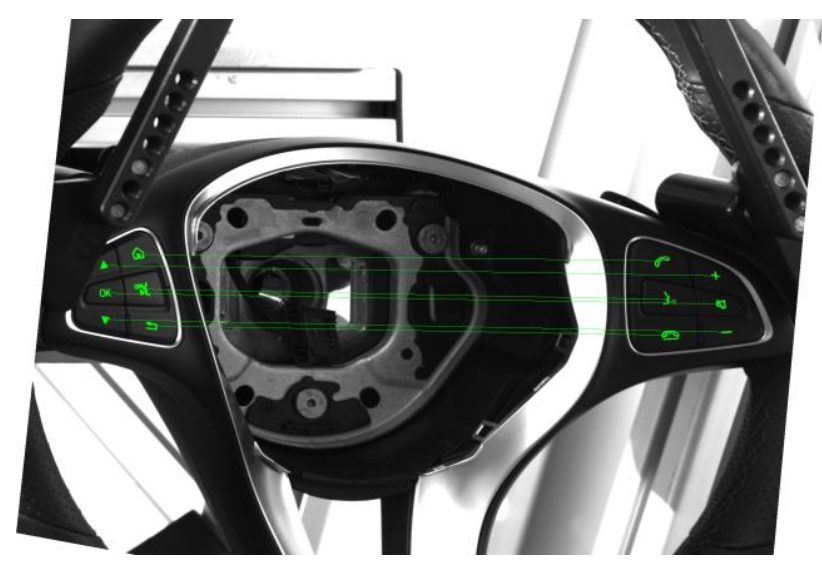

Figure 13. Point-to-point matching using several pairs of points to compute the slope in the rectified image

\section{CONCLUSIONS}

The obtained results have shown that computer vision systems based on automatic image analysis can fulfil the requirements of accuracy, contact-free measurements and real time analysis, indispensable preconditions for fully automatic inspection processes in the industrial production environment. Especially the point-to-point matching has confirmed its suitability with a detection rate of $100 \%$, an angle accuracy of $\pm 0.24^{\circ}$ and a processing time of $242 \mathrm{msec}$ including image acquisition. Only the 3D shape-based matching led to insufficient results containing several gross errors due to the spatial tolerances of the manufactured steering wheels compared to the CAD models.

In the future - for a general applicability of this approach - an extension to other types of steering wheels has to be carried out which can be obtained without any problem due to the availability of appropriate 3D construction plans (CAD models) as a standard of industrial productions. The only precondition is the existence of distinctive elements on the steering wheel. Therefore, in the near future the nowadays common mechanical balances may be replaced by computer vision systems.

\section{REFERENCES}

DSA, 2016. DSA Daten- und Systemtechnik GmbH, http://www.dsa.de [Accessed 10. March 2016]

Dürr, 2016a. Product Info "End of Line Testing". Dürr Assembly Products $\mathrm{GmbH}$, Püttlingen, Germany, http://www.durr-assembly.com/ileadmin/user_upload/ assembly/PDF_Artikel/Bro-End-of-line-en.pdf [Accessed 07. April 2016]

Dürr, 2016b. http://www.durr.com [Accessed 10. March 2016]
Fan, X., Wang, X. and Y. Xiao, 2014. A Shape-based Stereo Matching Algorithm for Binocular Vision. IEEE International Conference on Security. Pattern Analysis and Cybernetics (SPAC), pp. 70-74.

Gunasekaran, S., 1996. Computer vision technology for food quality assurance. Trends in Food Science \& Technology, Volume 7, no. 8, pp. 245-256.

HALCON, 2015a. Quick Guide, Edition 7a. MVTec Software $\mathrm{GmbH}$, München.

HALCON, 2015b. Solution Guide II-B Matching, Edition 3a. MVTec Software GmbH, München.

HALCON, 2015c. Solution Guide III-C 3D Vision, Edition 7a. MVTec Software GmbH, München.

HALCON, 2016. http://www.halcon.com/ [Accessed 07. April 2016]

Haniff, H.M., Sulaiman, M., Shah, H.N.M. and L. Teck, 2011. Shape-Based Matching: Defect Inspection of Glue Process in Vision System. IEEE Symposium on Industrial Electronics and Applications (ISIEA), pp. 53-75.

IDS, 2016. https://de.ids-imaging.com/ [Accessed 07. April 2016]

Molleda, J., Usamentiaga, R., García, D.F., Bulnes, F.G., Espina, A., Bassiru, D. and L.N. Smith, 2013. An improved 3D imaging system for dimensional quality inspection of rolled products in the metal industry. Computers in Industry, 64, pp. 1186-1200.

Reinbacher, C., Rüther, M. and H. Bischof, 2010. Pose Estimation of Known Objects by Efficient Silhouette Matching. IEEE 20th International Conference on Pattern Recognition, pp. 1080-1083.

Rodrigues, J.J., Kim, J.S., Furukawa, M., Xavier, J., Aguiar, P. and T. Kanade, 2012. 6D pose estimation of textureless shiny objects using random ferns for bin-picking. IEEE/RSJ International Conference on Intelligent Robots and Systems (IROS), pp. 3334-3341.

Ulrich, M., Steger, C. and A. Baumgartner, 2003. Real-time object recognition using a modified generalized Hough transform. Pattern Recognition, Vol. 36, pp. 2557-2570.

Ulrich M., Wiedemann, C. and C. Steger, 2009. CAD-Based Recognition of 3D Objects in Monocular Images. ICRA'09, Proceedings of the 2009 IEEE International Conference on Robotics and Automation, pp. 2090-2097.

Xu, X., Zhang, X., Han, J and C. Wu, 2008. HALCON Application for Shape-Based Matching. Industrial Electronics and Application, ICIEA, 3rd IEEE Conference, pp. 2431-2434. 